\title{
Directional Screens
}

\author{
Michal Piovarči ${ }^{1,2} \quad$ Michael Wessely ${ }^{1,3} \quad$ Michał Jagielski $^{1} \quad$ Marc Alexa $^{4}$ \\ Wojciech Matusik ${ }^{5}$ Piotr Didyk Di,2 $^{1,2}$
}

${ }^{1}$ Saarland University, MMCI $\quad{ }^{2}$ MPI Informatik $\quad{ }^{3}$ Inria, Univ. Paris-Sud, CNRS, Université Paris-Saclay $\quad{ }^{4}$ TU Berlin $\quad{ }^{5}$ MIT CSAIL
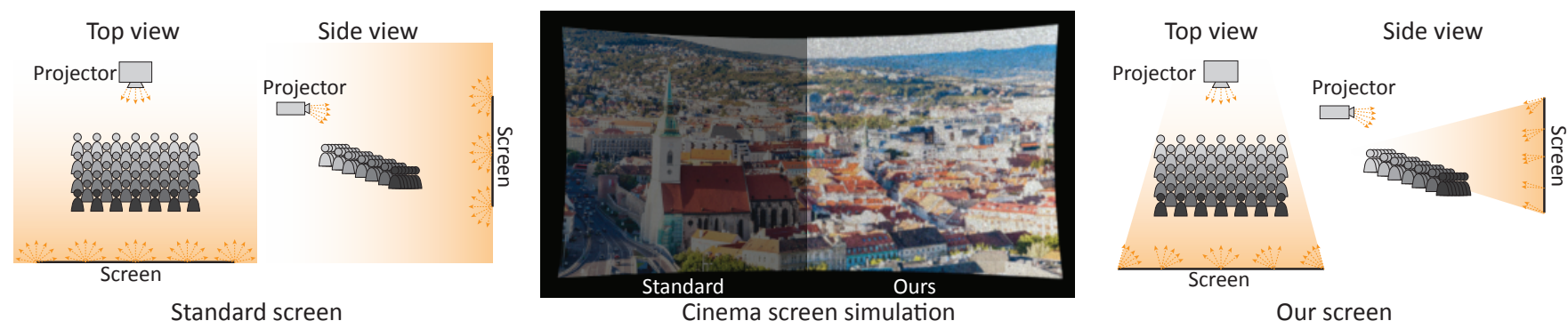

Figure 1: Current screens reflect significant amounts of light into regions where the audience is not expected (left). Reflecting the light toward the ceiling, floor or walls is unnecessary and causes energy loss. Our design enables a fine control over the reflectance properties of the screen surface (right), which provides better energy efficiency, brightness, and contrast.

\begin{abstract}
The goal of display and screen manufacturers is to design devices or surfaces that maximize the perceived image quality, e.g., resolution, brightness, and color reproduction. Very often, a particular viewer location is not taken into account, and the quality is maximized across all viewing directions. This, however, has significant implications for energy efficiency. There is usually a very wide range of viewing directions (e.g., ceiling, floor, or walls) for which the displayed content does not need to be provided. Ignoring this fact results in energy waste due to a significant amount of light reflected towards these regions. In our work, we propose a new type of screen - directional screens, which can be customized depending on a specific audience layout. They can provide up to 5 times increased gain when compared to high-gain screens and up to 15 times brighter reflection than a matte screen. In addition, they provide uniform brightness across all viewing directions, which addresses the problem of "hot-spotting" in high-gain screens. The key idea of our approach is to build a front-projection screen from tiny, highly reflective surfaces. Each of these surfaces is carefully designed so that it reflects the light only towards the audience. In this paper, we propose a complete process for designing and manufacturing such screens. We also validate our concept in simulations and by fabricating several fragments of big screens.
\end{abstract}

Permission to make digital or hard copies of all or part of this work for personal or classroom use is granted without fee provided that copies are not made or distributed for profit or commercial advantage and that copies bear this notice and the full citation on the first page. Copyrights for components of this work owned by others than the author(s) must be honored. Abstracting with credit is permitted. To copy otherwise, or republish, to post on servers or to redistribute to lists, requires prior specific permission and/or a fee. Request permissions from permissions@acm.org.

SCF '17, fune 12-13, 2017, Cambridge, MA, USA

(c) 2017 Copyright held by the owner/author(s). Publication rights licensed to Association for Computing Machinery.

ACM ISBN 978-1-4503-4999-4/17/06 . \$ \$15.00

https://doi.org/10.1145/3083157.3083162

\section{CCS CONCEPTS}

- Hardware $\rightarrow$ Displays and imagers; • Computing methodologies $\rightarrow$ Reflectance modeling;

\section{KEYWORDS}

computational fabrication, reflectance, energy efficiencent screens

ACM Reference format:

Michal Piovarči, Michael Wessely, Michał Jagielski, Marc Alexa, Wojciech Matusik, and Piotr Didyk. 2017. Directional Screens. In Proceedings of SCF '17, Cambridge, MA, USA, fune 12-13, 2017, 10 pages.

https://doi.org/10.1145/3083157.3083162

\section{INTRODUCTION}

Continuous demand for higher quality image reproduction forces display manufacturers to refine their designs constantly. This results in a continuous increase of spatial resolution, contrast, color gamut, and frame rate. The process is also stimulated by improving the quality of acquisition apparatus, i.e., high resolution and high dynamic range cameras impose new requirements on display devices regarding faithful reproduction of the content. While many hardware and software solutions for improving display quality exist, the efficient use of emitted light is very often overlooked and the increase in display quality comes at the price of energy efficiency. For instance, a display system usually provides high-quality brightness and color reproduction independently of the viewer position. This, however, is often unnecessary, which becomes even more evident in the movie theater scenario, where the audience layout is precisely defined. In such cases, it is sufficient to provide high image quality only for positions where the audience is expected and avoid emitting light in other directions, e.g., the walls or ceiling.

An efficient use of light can also lead to brighter screens. This becomes very important for 3D movie theaters, where the overall 
brightness is reduced to roughly $20 \%$ of its initial value [Cowan 2008]. Given that the standard brightness for $2 \mathrm{D}$ cinema is $14-16 \mathrm{fL}$ (footlambert) $\left(48-55 \mathrm{~cd} / \mathrm{m}^{2}\right)$, using the same projector for 3D applications results in around $3 \mathrm{fL}\left(10 \mathrm{~cd} / \mathrm{m}^{2}\right)$. This has significant implications for perceived quality. In such conditions, human perception operates on the boundary between mesopic and photopic vision, where spatial acuity, perceived contrast, depth perception, and color vision are significantly affected [Bartleson and Breneman 1967; Lit 1959; Shin et al. 2004]. A natural solution for the problem is to provide brighter projectors [Schuck and Sharp 2012]. This, however, leads to significantly increased operation costs as brighter projection lamps consume more energy, are more expensive, and burn out more quickly. This can potentially quadruple the annual maintenance cost of a single projector [Harkness Screens 2014]. In the context of screen designs, the problem can be overcome by high-gain screens which boost the brightness, but suffer from hotspotting, i.e., a brightness fall-off towards the boundaries, due to their significant specular component.

Inspired by recent developments in computational techniques for manufacturing complex optical components [Damberg and Heidrich 2015; Tompkin et al. 2013; Weyrich et al. 2009], in this work, we propose a new way of designing and fabricating projection screens. The key idea is to produce large surfaces with prescribed, spatially-varying reflectance properties that reflect light only towards the audience, (Figure 1). To this end, we propose to build the surfaces from small geometrical components (microgeometries) made of a highly reflective material. Each microgeometry has a prescribed normal distribution so that it reflects the light uniformly in a precisely defined direction range. To compute our microgeometries, we adapt a convex optimization, such that it guarantees the exact normal distribution and tileability of the shapes. As our screens are made of a highly reflective surface, they preserve polarization, and therefore can be used in 3D movie theaters. Our design process is evaluated using simulations, as well as capture of several manufactured screen parts. Besides designing standard screens, e.g., for movie theaters, our technique can be used in more challenging cases. We demonstrate this by producing a screen which reflects the light into two disjoint regions.

\section{PREVIOUS WORK}

To maximize light efficiency, an optimal screen should reflect light only towards the audience. As the required angular light coverage varies across the screen (Figure 1), the optimal solution requires producing a surface with spatially-varying reflectance properties (SVBRDF). This problem is not only relevant for screen design, but it has also been broadly researched in the context of appearance reproduction. In this section, we will discuss the work from both fields. Our technique is also related to reflector designs, where the shape of a highly reflective surface is optimized to reflect light in the desired direction. We refer the reader to the following survey for discussion of these techniques [Patow and Pueyo 2005].

Screen Design. Over last several decades, screen design evolved from a simple, Lambertian-like surface (i.e., a matte screen), which reflects light uniformly in all directions, to more sophisticated ones, where both the material as well as the geometrical structure of the screen are carefully designed to maximize the portion of light reflected towards the audience. The simplest solutions involve covering the screen surface with a semi-glossy coating [Chandler and De Palma 1968]. Such screens are usually characterized by their gain factor, which is a ratio of the light reflected by the screen as compared to the light reflected from a matte screen. Although they provide a better efficiency, they suffer from a "hot-spot" effect, i.e., the observed brightness is uniform neither across the screen nor at different viewing locations. Additionally, the highest gain is achieved only for a center position in the audience [Richards and Schnuelle 2010]. The problem can be reduced to a certain extent by using a curved screen geometry [Berggren and Carignan 1967]. A finer control over the screen reflectance properties can be achieved by modifying the local geometry of a highly reflective surface. Although several commercial solutions have been proposed [Cobb et al. 1980; Hockley and Pawluczyk 1991; Sun 2001; Van De Ven 1990], it is unclear how optimal these solutions are, as there is not enough detail to faithfully reproduce or simulate the designs and no qualitative evaluation is provided. One of the most recent solutions in this area was proposed by Coleman et al. [2011]. Similarly to us, they take a purely geometrical approach, i.e., they build the screen using a mirror-like surface and control the reflectance properties by careful design of the screen surface. They proposed to build the surface out of small kernel-like shapes which are designed according to desired reflectance. They show how to tile such shapes to obtain the entire screen surface. Although they can vary the shapes locally, their method assumes that the shapes are axially symmetric. This limits their flexibility in adjusting the reflectance properties locally. In contrast, our technique is capable of producing surfaces with reflectance properties perfectly matching an arbitrary audience layout. An alternative approach is to modify the direction of the light via microscopic optical diffusers [Luminit 2016; Photonics 1980]. Recently, [Crystal Screens 2016] developed a new kind of reflective holographic screen with high gain (2.5) and a wide viewing angle $\left(120^{\circ}\right)$. However, the screen has limited size, up to $2.4 \times 1.3$ meters, and it is unclear how it can be used in cinemas where the average screen size is $16 \times 7$ meters.

Appearence Reproduction. Recent developments in computational fabrication enabled fabrication of objects with prescribed reflectance properties (i.e., BDRF and SVBRDF). To this end, several approaches have been proposed. Weyrich et al. [2009] presented one of the first techniques to rely on a purely geometrical interpretation of BRDF, so-called microfacets theory [Blinn 1977; Cook and Torrance 1982]. They proposed to build a surface from tiny, highly reflective "micromirrors" whose normal distribution matches the prescribed BRDF. The arrangement of these mirrors is computed using an expensive simulated annealing optimization. In contrast, our method is based on efficient convex optimization which allows us to compute much larger surfaces required for cinema screens. Rouiller et al. [2013] has recently applied a similar idea. Instead of producing a surface with a desired facet arrangement, they proposed to compute tiny shapes - domes, which encode the BRDF properties. Later, they are placed on objects to affect their appearance. In contrast to the previous solution, this method does not produce a continuous surface. Recently, Levin et al. [2013] considered the problem on a much smaller scale. Instead of using the geometrical interpretation of BRDF, they showed how to account for wave effects and 


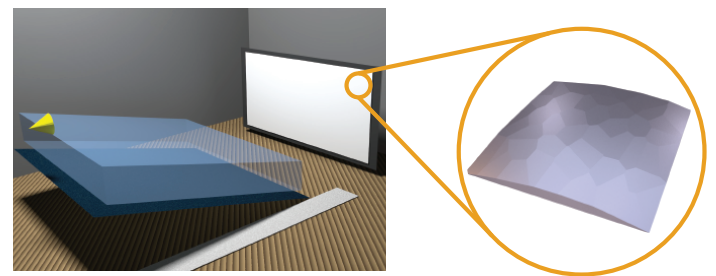

Define Cinema Arrangement Compute Surface Geometries

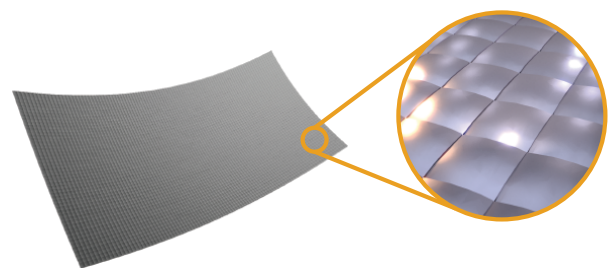

Generate Screen Shape and Surface

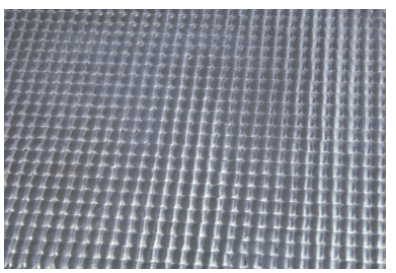

Manufacture Screen

Figure 2: Display generation overview: We start with the definition of the screen, the projector, and the audience. Based on this knowledge we uniformly sample the screen and for each position generate a microgeometry. These geometries are tiled and their heights adjusted to form the final screen surface. The screen surface is manufactured from aluminum on a CNC milling machine.

change the appearance of the surface by controlling diffraction effects. They demonstrated impressive, high-resolution results with minimal feature size as small as 2-3 $\mu \mathrm{m}$. However, due to high fabrication costs, they were able to demonstrate only small samples. Matusik et al. [2009] proposed a different method for controlling and manufacturing material appearance. Instead of modifying local microgeometry, they suggested to use inks with different reflectance properties, which when mixed, provide a broad range of spatially-varying BRDFs. Such an approach can be also combined with micro-facet techniques, where both geometry and inks are optimized to obtain the desired appearance [Lan et al. 2013; Malzbender et al. 2012]. Instead of optimizing micro-geometry, it is possible to choose it from a precomputed database [Wu et al. 2013]. In contrast to these methods, we are the first to show how to efficiently generate spatially-varying BRDF for very large surfaces. Unlike many methods mentioned above, our technique is capable of producing exact reflectance properties under the assumption that the surface behaves as a mirror.

Light-Efficient Projectors. Recently, there has also been a significant effort in increasing light efficiency of projectors to enable high-dynamic-range capabilities. Hoskinson et al. [2010] proposed a system where instead of blocking light from an LCD panel, an additional digital micromirror device (DMD) reallocates the light to bright regions. The solution has been improved recently by replacing the DMD with a phase modulator [Damberg et al. 2016]. These solutions are complementary to our efforts aiming at producing a better screen and can be used together in one projection system.

\section{DISPLAY DESIGN}

We take a geometrical approach for creating a screen surface and rely on the microfacet theory [Cook and Torrance 1982; Heitz 2014; Torrance and Sparrow 1967]. The overview of our technique is presented in Figure 2. To design the geometry of the screen surface, our method takes as an input the position and the size of the screen, projector location, and the audience. Without losing generality, we assume that the audience is defined as one or more polyhedrons which enclose the areas where viewers are expected. First, we consider a problem of computing small local shapes that for each location on the screen reflect light according to the audience description (Section 3.1). We formulate this problem by adopting a convex optimization for reconstruction of polyhedrons from Extended Gaussian Images [Little 1983]. In the next step, we combine local geometries into one surface (Section 3.2). Elevation of each shape is adjusted to minimize masking and shadowing. The nonuniform reflectance characteristic of our screen requires an additional content adjustment. We describe it in Section 3.3. We validate our designs in simulations and by manufacturing smaller screen sections from aluminum using a CNC milling machine (Section 4).

\subsection{Microgeometry Design}

The goal of the local geometry is to reflect incoming projector light only in directions where the audience is expected. Additionally, we should assure uniform luminance of the screen across different viewing directions. In other words, we want to create a microgeometry which acts as a diffuse surface, but only in the range of directions specified by the audience layout. In the regime of microfacet theory, producing such a surface is equivalent to designing a microgeometry composed of highly reflective facets whose normal distribution fulfills the requirement. We do this in two steps. First, we derive a set of facets defined by their normals and areas. Then we construct a convex, tileable shape from these facets.

Facet definition. In previous work, microfacet geometry was usually derived assuming that the size of every facet is equal. A set of microfacets was generated by sampling the desired normal distribution [Weyrich et al. 2009]. In our work, we propose to first derive facet normals and then adjust their areas to reproduce the desired BRDF. To this end, for a given location on the screen $\mathbf{x}$, we construct a set of directions $\mathbf{V}_{\mathbf{X}}$ in which the projected light should be reflected. We uniformly sample all directions which lie in the half-space adjacent to the screen surface and include directions that intersect with the audience. For each direction $\mathbf{o}_{i} \in \mathbf{V}_{\mathbf{X}}$ we define a corresponding normal vector of the facet $\mathbf{h}_{i} \in \mathbf{H}_{\mathbf{x}}$ as $\mathbf{h}_{i}=\left(\mathbf{o}_{i}+\mathbf{i}\right) /\left\|\mathbf{o}_{i}+\mathbf{i}\right\|$, where $\mathbf{i}$ is the direction of incident light the direction towards the projector (Figure 3).

To complete the definition of the facets, we need to determine areas $a_{i}$ for each of them, which will define the resulting BRDF. Walter et al. [2007] presented an equation for specular microfacetbased BRDFs, where the reflectance of the surface is defined using a microfacet normal distribution function $D$ :

$$
\rho(\mathbf{i}, \mathbf{o})=\frac{F(\mathbf{i}, \mathbf{h}) G(\mathbf{i}, \mathbf{o}, \mathbf{h}) D(\mathbf{h})}{4|\mathbf{i} \cdot \mathbf{n}||\mathbf{o} \cdot \mathbf{n}|} .
$$

Additional terms $F$ and $G$ denote Fresnel and masking-shadowing terms, respectively, and the operator $|\cdot|$ denotes the dot product 


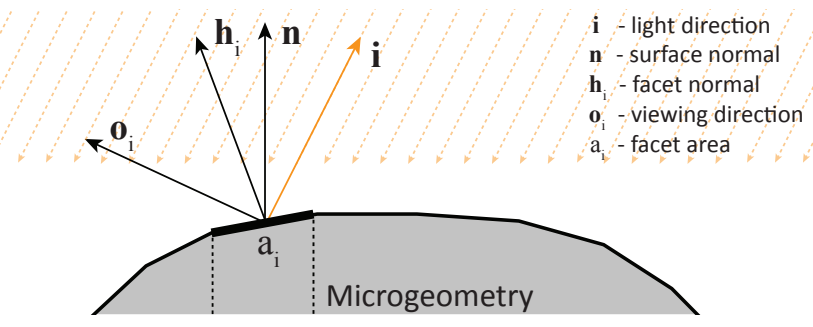

Figure 3: Notation used in our derivation.

between two vectors. For a comprehensive derivation, we refer the reader to [Heitz 2014]. In our case, we want to design a surface that has Lambertian reflectance in a range of directions corresponding to the audience viewing directions and does not reflect light outside. Therefore, we seek a microgeometry such that $\rho(\mathbf{i}, \mathbf{o})=\rho$ for all $\mathbf{o} \in \mathbf{V}_{\mathbf{x}}$, and $\rho(\mathbf{i}, \mathbf{o})=0$ for $\mathbf{o} \notin \mathbf{V}_{\mathbf{x}}$. Note that $D(\mathbf{h})$ is in fact a normalized area of a facet with a normal $\mathbf{h}$; therefore, we can use Equation 1 to directly define the areas $a_{i}$ as:

$$
a_{i}=\frac{4 \rho|\mathbf{i} \cdot \mathbf{n}|\left|\mathbf{o}_{i} \cdot \mathbf{n}\right|}{F\left(\mathbf{i}, \mathbf{h}_{i}\right) G\left(\mathbf{i}, \mathbf{o}_{i}, \mathbf{h}_{i}\right)} .
$$

In our microsurface derivation, we can omit masking-shadowing term $G$ as we construct convex shapes for which the term is constant. Furthermore, we assume that the light direction does not change for small microgeometries. Therefore, $|\mathbf{i} \cdot \mathbf{n}|$ is also constant. Because we are interested in reconstructing the microgeometry up to a scalar, all constant factors can be omitted and Equation 2 simplifies to:

$$
a_{i}=\frac{\left|\mathbf{o}_{i} \cdot \mathbf{n}\right|}{F\left(\mathbf{i}, \mathbf{h}_{i}\right)} .
$$

We wish to generate the geometry of small patches with the desired set of facet normals $\left\{\mathbf{h}_{i}\right\}$ and areas $\left\{a_{i}\right\}$. The patches also should tightly cover the screen surface. Consequently, we opted for patches with squared bases. As the desired sets of normals and areas that should form a microgeometry do not guarantee that the resulting shape will have a squared base, we add four additional faces. We call them side faces as they are perpendicular to the screen plane and form a rectangle on the screen surface. As we will demonstrate in this section, by optimizing the areas of the additional faces as well as their distances from the center of the microgeometry, it is possible to guarantee a perfect square-shape of the base. To summarize, we wish to generate microgeometries that have:

- faces with normals $\mathbf{H}_{\mathbf{x}}=\left\{\mathbf{h}_{i}\right\}$ and corresponding areas $\mathbf{A}_{\mathbf{x}}=\left\{a_{i}\right\}$

- four side faces with normals $\pm \mathbf{r}, \pm \mathbf{s}$ and arbitrary but nonnegative areas $a_{\mathbf{r}_{0,1}, \mathbf{s}_{0,1}}$, where the face normals are chosen orthogonal to each other and to the screen normal, i.e., $\mathbf{s} \cdot \mathbf{r}=0, \mathbf{s} \times \mathbf{r}=\mathbf{n}$,

- no other faces with normals in the positive half-space of the screen surface.

We base our construction on Minkowski's theorem on convex polyhedra with prescribed normals and areas [Minkowski 1989], which says that a polyhedron exists and is unique if the area-weighted face normals sum to zero. Given the constraints, this suggests requiring that the areas for side faces are identical, i.e., $a_{\mathbf{r}_{0}}=a_{\mathbf{r}_{1}}$ and $a_{\mathbf{s}_{0}}=a_{\mathbf{s}_{1}}$, and defining one additional face with area-weighted normal $-\sum_{i} a_{i} \mathbf{h}_{i}$ that serves as the base of our microgeometry.

Alexandrov [2005] and Little [1983] have found a variational principle for the problem when all areas are given. It is instructive to introduce their idea for solving the more general problem we have at hand here. Notice that the product of area and face normal $a_{i} \mathbf{h}_{i}$ is the gradient of the volume of a polyhedron relative to a face with normal $\mathbf{h}_{i}$ and area $a_{i}$. The fact these products sum to zero suggests that the polyhedron has extremal volume under variation of the distances $l_{i}$ of the facets to the origin (i.e., a face $i$ is contained in the plane $\mathbf{h}_{i} \cdot \mathbf{x}=l_{i}$ ). Alexandrov has found that the right constraint is to fix the sum of the area weighted distances $\sum_{i} a_{i} l_{i}=1-$ with this constraint the polyhedron with maximal volume has the desired face areas. On the other hand Little suggests to fix the volume of the polyhedron and minimize $\sum_{i} a_{i} l_{i}$. Both formulations lead to an efficient convex optimization. In our implementation we decided to adapt the algorithm proposed by Little.

In our setting we also need to consider the side faces with areas $a_{\mathbf{r}}, a_{\mathbf{S}}$ (which are identical for opposing sides) and distances $l_{\mathbf{r}_{0,1}, \mathbf{s}_{0,1}}$. Let $\mathbf{l}=\left(l_{0}, \ldots\right)$ be the vector of distances. Together with the fixed facet normals $\left\{\mathbf{h}_{i}\right\}$, it defines the polyhedron, and therefore, its volume $V(\mathbf{l})$, as well as the areas of the facets $\left\{A_{i}(\mathbf{l})\right\}$. In addition to the volume being constant, we ask that the side faces intersect the base plane in a square and the free areas $a_{\mathbf{r}}, a_{\mathbf{s}}$ add up to a constant. More formally, we have the following constraints:

$$
1=V(\mathbf{l}), \quad l_{\mathbf{r}_{0}}+l_{\mathbf{r}_{1}}=l_{\mathbf{s}_{0}}+l_{\mathbf{s}_{1}}, \quad c=a_{\mathbf{r}}+a_{\mathbf{s}},
$$

where $c$ is an additional constant fixed during the optimization. With these notations, the functional to be minimized is

$$
\sum_{i} a_{i} l_{i}+a_{\mathbf{r}}\left(l_{\mathbf{r}_{0}}+l_{\mathbf{r}_{1}}\right)+a_{\mathbf{s}}\left(l_{\mathbf{s}_{0}}+l_{\mathbf{s}_{1}}\right) \text {. }
$$

Comparing this to the original formulation, we have added two free degrees of freedom, i.e., the area variables $a_{\mathbf{r}}, a_{\mathbf{s}}$, but also two new constraints (Equation 4). As a results, the degrees of freedom still match the number of constraints, and the solution is unique up to translation. We fix this last degree of freedom by setting the center of the polyhedron to be in the world's origin.

We iteratively minimize Equation 5 as a function of 1 , under the constraints of Equation 4. As suggested by Little [1983] each step is taken along the gradient of $\sum_{i} a_{i} l_{i}$ restricted to the hyperplane perpendicular to the gradient of the volume. This ensures that each step does not deviate significantly from the constraint $V(\mathbf{l})=1$. Note that we do also need to optimize for $l_{\mathbf{r}_{0,1}}, l_{\mathbf{s}_{0,1}}$. The reason is that $\mathbf{l}$ influences the areas $A(\mathbf{l})_{\mathbf{r}_{0,1}}, A(\mathbf{l})_{\mathbf{s}_{0,1}}$ of the side faces, which are supposed to be the same for opposite sides. So the variables $a_{\mathrm{r}, \mathrm{s}}$ need to be adjusted, which we do by projecting the current values $A(\mathbf{l})_{\mathbf{r}_{0,1}}, A(\mathbf{l})_{\mathbf{s}_{0,1}}$ orthogonally onto the affine subspace:

$$
\begin{aligned}
0 & =A(\mathbf{l})_{\mathbf{r}_{0}}-A(\mathbf{l})_{\mathbf{r}_{1}} \\
0 & =A(\mathbf{l})_{\mathbf{s}_{0}}-A(\mathbf{l})_{\mathbf{s}_{1}} \\
2 c & =A(\mathbf{l})_{\mathbf{r}_{0}}+A(\mathbf{l})_{\mathbf{r}_{1}}+A(\mathbf{l})_{\mathbf{s}_{0}}+A(\mathbf{l})_{\mathbf{s}_{1}} .
\end{aligned}
$$

The constraint on the distances to the side faces is similarly enforced by projecting onto the linear subspace given by the constraint $l_{r_{0}}+$ $l_{\mathbf{r}_{1}}-l_{\mathbf{s}_{0}}-l_{\mathrm{s}_{1}}=0$. This formulation guarantees that the bounding box of each microgeometry is a square. The only criterion for generation of tileable geometries is that the side areas $a_{\mathrm{r}}, a_{\mathrm{s}}$ are sufficiently 
large, which can be enforced by appropriately choosing c. During our experiments we have found out that setting $c$ proportionally to $\sum\left\{A_{i}(\mathbf{l})\right\}$ is sufficient for generating all examples described in the paper. The pseudo-code of our method is described in Algorithm 1. Figure 4 shows snapshot of a microgeometry during optimization. At each iteration the geometry is tileable and the top faces change their area until they converge to the desired shape.
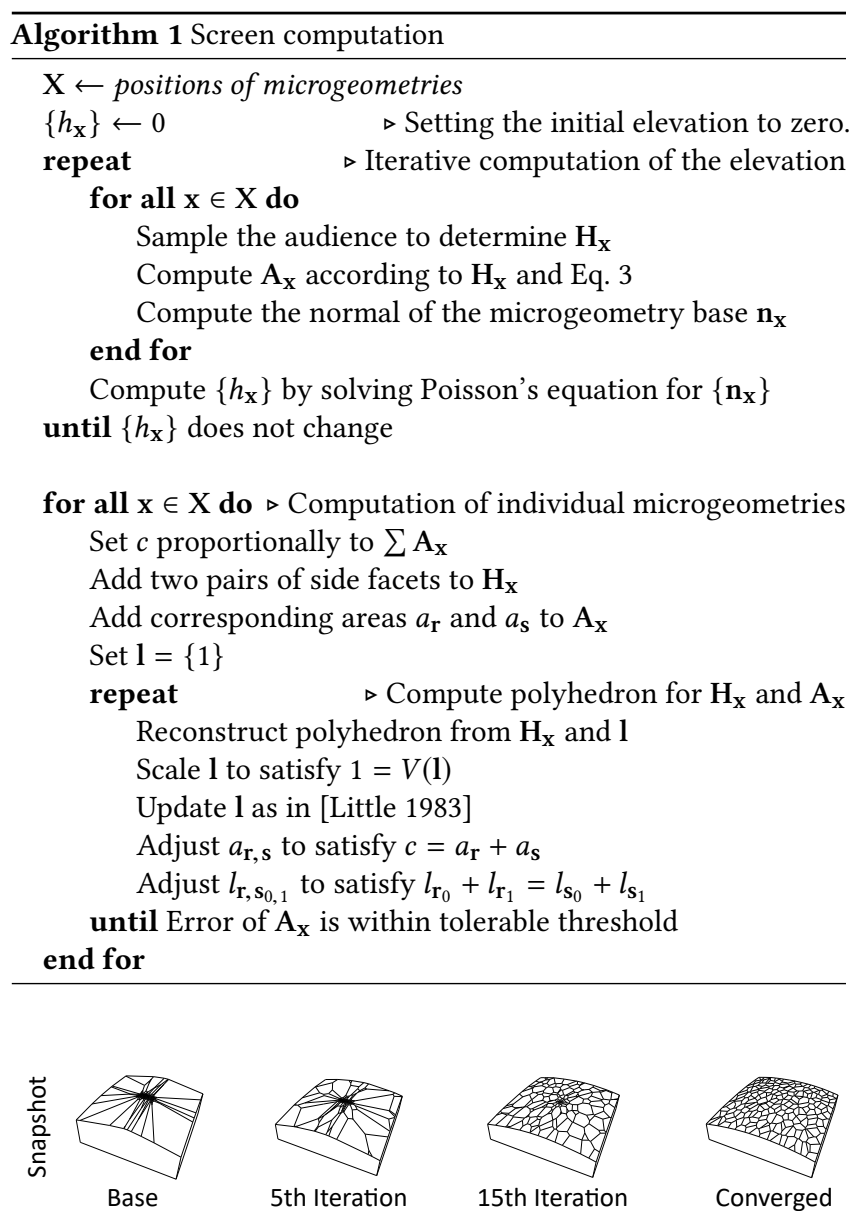

Figure 4: Snapshots of exemplar microgeometry during optimization.

\subsection{Screen Design}

We demonstrated how to compute a microgeometry for an arbitrary location $\mathbf{x}$ on the screen surface. To build the entire screen, every location on its surface must be covered by microgeometry. This can be done by dividing the screen into a uniform grid. Then for each grid cell, a microgeometry can be computed according to our method (Algorithm 1). The microgeometries are generated up to scale. Therefore, we have to rescale each microgeometry before it is placed on the screen surface.

The screen surface computed using our optimization may have discontinuities between neighboring microgeometries, which can lead to masking and shadowing effects (Figure 5). To address this problem, we align the bases of microgeometries such that they form a smooth surface, minimizing the discontinuities. This is done by finding an appropriate elevation of each base by solving a discrete Poisson's equation, similarly to [Weyrich et al. 2009]. As we change the elevation, the location of each microgeometry with respect to the audience and the projector changes. Therefore, we have to interleave the solve of the Poisson's problem with updating the normals of the microgeometry bases. In practice, computation of the smooth screen surface requires several iterations of sequentially solving the Poisson's problem and updating the normals. The process is very fast as the reconstruction of individual microgeometries is not required at this stage.
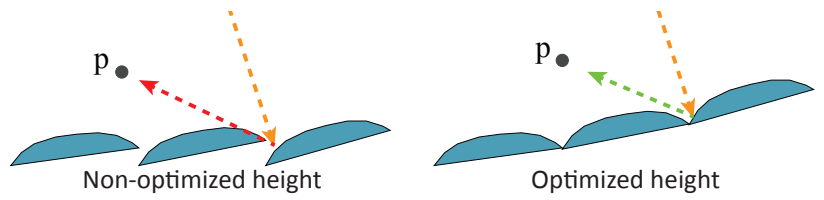

Figure 5: When the screen surface has discontinuities, shadowing or masking can occur. In this case, a light ray coming from a projector (yellow) should be reflected towards position p. A discontinuous screen surface may shadow the reflected ray (left). This does not happen when the surface is continuous (right).

\subsection{Content Preparation}

Our screens are designed so that they reflect light uniformly, i.e., the brightness of any particular location on the screen does not depend on viewing direction. However, because the light reflected from the different parts of the screen spans different solid angles, uniform illumination of the screen will not produce uniform brightness across the screen. The projector needs to be calibrated before an image is displayed. Intuitively, the areas of the screen which reflect the light in smaller angles should receive proportionally less light. The amount of light reflected in each direction should be proportional to the cosine of the viewing angle. Consequently, the total amount of light required at a given location $\mathrm{x}$ of the screen can be calculated as:

$$
L(\mathbf{x})=\int_{\Omega^{+}} \cos \theta \cdot V(\Psi) \cdot d \omega \Psi,
$$

where function $V$ has value 1 when the direction $\Psi$ intersects with the audience volume and 0 otherwise. $\theta$ is the angle between the surface of the screen and $\Psi$. Depending on the definition of the audience, it might be difficult to compute $L$ analytically. In our work, we computed it numerically. The most straightforward way of applying the compensation is to apply a darkening mask as a digital filter on the projector or as a filter inserted before the projector lens. This will not lead to the most efficient light use. However, we demonstrate that even with such a simple approach we can achieve a significant efficiency boost. More recently, new designs of HDR projector systems have been presented [Damberg et al. 2016; Hoskinson et al. 2010]. Our screens are perfectly suitable for these solutions that can simply redirect the light illuminating each part of the screen according to Equation 7. In the next sections, we report results for both compensation methods. 


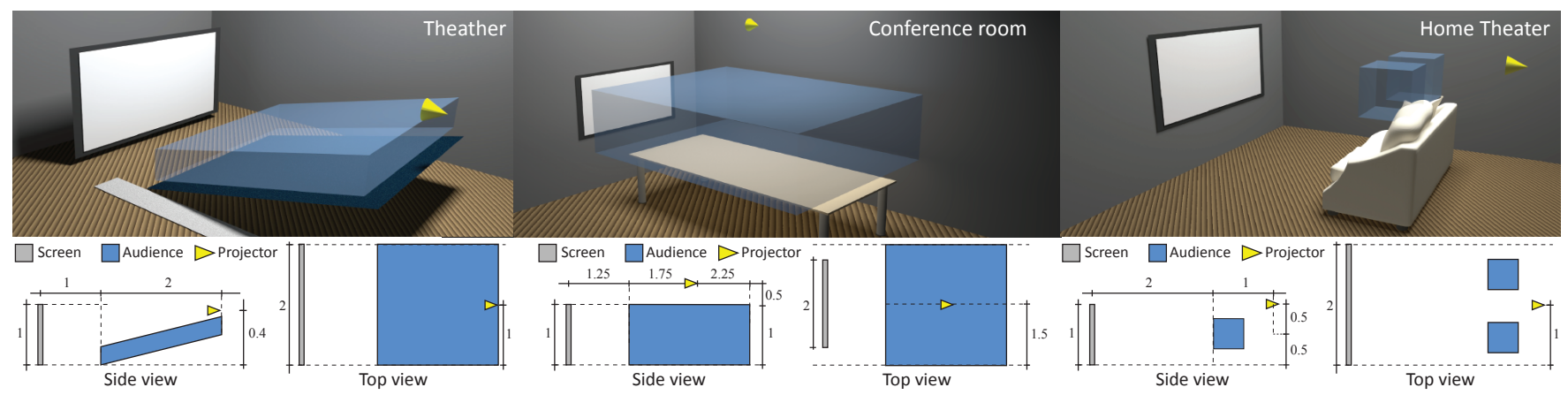

Figure 6: We consider three installations in this paper. The first and the second setup depict one audience, in a theater and in a conference room. The third installation conceives a home theater with two separate audience spaces, for each viewer. Note that the units are left undefined as the design is general and can be scaled to different sizes. This also does not affect our screen computation.

\section{RESULTS}

We evaluated our technique by both simulating (Section 4.2) several screen designs and fabricating (Section 4.3) their parts. Even though producing complex mirror surfaces is possible, we were limited by rather low-cost methods of fabricating our prototypes and chose to use a 3-axis $\mathrm{CNC}$ machine to mill our prototypes from aluminum. This allowed us to obtain highly glossy surfaces, but not perfect mirrors. On one hand, this worsens our results, but on the other hand, it validates the benefits of our design in cases when the fabrication is not perfect. We demonstrate in this section that even with such deviations from a perfect mirror-like BRDF we can achieve favorable results, i.e., brighter and more uniform screens, when compared to matte and high gain screens. Consequently, we also present simulation results obtained using the BRDF of polished aluminum. Despite the limitations of our low-cost manufacturing process, we argue that, in practice, surfaces that are much closer to mirrors can be achieved.

\subsection{Technical Details}

To validate our model we consider three screen designs. The first two are common use cases: a theater (Figure 6a) and a conference room (Figure 6b). To push our technique to the limits and demonstrate novel applications, we also present a home theater (Figure 6c) which creates a split view, i.e., the content can be observed only from two disjoint viewing volumes. Although such setups are not common, we believe they can find applications in custom visualization setups.

We generated the screens using microgeometries with 200 normals. This was determined based on the geometry size that we were able to simulate and manufacture. Since our screens are manufactured from polished aluminum, the Fresnel term is constant and the area of each microfacet was solely determined by the viewing angle. To optimize the trade-off between geometry precision and the computational time, we terminated the optimization when there was no facet whose area deviated by more than $1 \%$ from the desired value. The size of the microgeometries plays an important role. The images produced by our screen are composed of tiny reflections spaced by the distance equal to the size of one microgeometry.
Therefore, the size of the microgeometry should be small enough to make individual reflections invisible for a human eye but also big enough to enable fabrication and to avoid diffraction effects. In our simulations and physical prototypes, we used microgeometries with a width of $4 \mathrm{~mm}$. This corresponds to the size of one pixel in an average $4 \mathrm{~K}(4096 \times 2160)$ cinema. Note that the images produced with our screens are similar to those produced by big LED video walls where the size of LEDs is significantly smaller than the spacing between them.

All computations were run on Intel Xeon Processor E5-1620 v3. A total of $125 \mathrm{k}$ microgeometries were computed for each screen. An average microgeometry was computed in approximately 2 seconds, and the computation of the whole screen took roughly 6 hours. We analyzed the convergence of our square microgeometry computation algorithm. We randomly sampled geometries across all three of our designs and plotted their convergence (Figure 7). Since we base our algorithm on convex optimization proposed by [Little 1983], the most computationally demanding step at each iteration is the reconstruction of the connectivity of the geometry. This is computed using a convex hull algorithm running in $O(n \log n)$. An average geometry converges in approximately 50 iterations.

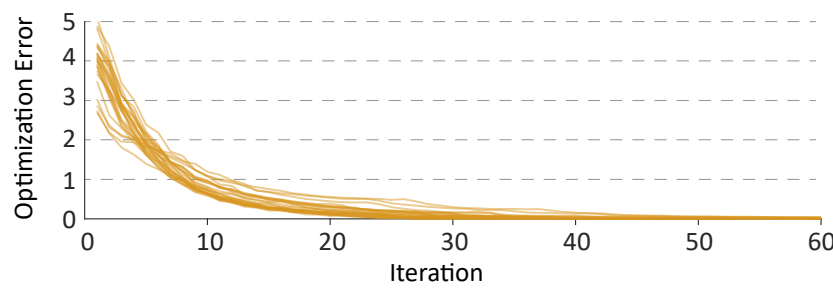

Figure 7: Convergence plot of randomly selected microgeometries.

\subsection{Simulations}

To simulate our screens, we used a physically-correct ray tracer that accounts for both shadowing and masking. For each screen, we created a virtual testing room by placing a screen, a projector, and 
an audience at their respective positions. Screens are modeled with a BRDF corresponding to polished aluminum. For each screen, we calculate the corresponding calibration mask (Figure 8) and model it as a modulation layer of the projector. In our simulation, we also accounted for human visual acuity. We assumed that the individual reflections from neighboring microgeometries are not resolved by the human visual system. To account for this, we filtered all our renderings using a Gaussian filter with a standard deviation corresponding to a visual angle spanned by one microgeometry seen from the middle of the audience. This is a realistic assumption for the movie theater case. Given a medium screen size $(15 \mathrm{~m})$ and the often mentioned optimal viewing angle of $36^{\circ}$ (THX), the resulting viewing distance is roughly $23 \mathrm{~m}$. The visual angle spanning one microgeometry used in our experiments $(4 \mathrm{~mm})$ is equal to 0.6 arcmin, which is below the smallest gap that an observer with $20 / 20$ vision can perceive. We evaluated the screens regarding their efficiency, as well as brightness uniformity across the screen and the audience. Finally, we simulated images shown on our screens. For full simulations, please refer to the supplemental video.

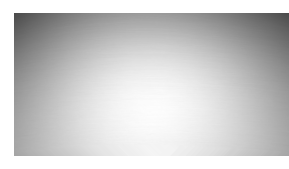

Theater

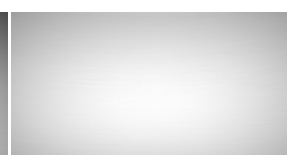

Conference Room

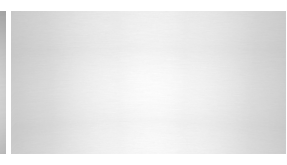

Home Theater
Figure 8: Calibration masks used to equalize the brightness across each screen.

We evaluated the distribution of the brightness provided by our screen across the audience by rendering illuminated screens from uniformly sampled locations within the audience. Since the split screen is symmetrical, we show results for the left view only. To limit aliasing problems, the views were computed in resolution $5 \times$ FullHD so that each microgeometry occupies more than one pixel. Next, we computed the average brightness for each view and plotted it as a function of position in the audience (Figure 9). As expected, due to the BRDF used in our experiment that slightly deviates from a perfect mirror reflection, the brightness provided by our directional screen is not perfectly uniform. The variation is, however, very small for the theater and conference room cases. The effect is more pronounced for the home theater. We attribute this to the relatively small audience size compared to the screen.

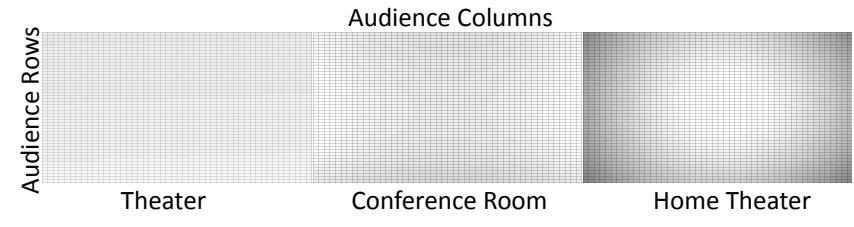

Luminance $0 \%$ $100 \%$

Figure 9: Screen brightness as a function of location within the audience.

Next, we compared our designs in terms of efficiency and brightness uniformity with matte and high gain screens. We did this by rendering a white patch on each screen according to ISO 3640-1976 [1978]. Figure 10 shows gain provided by each screen relative to a matte screen as a function of viewing angle. The results demonstrate that even with a glossy BRDF our screens provided better and more uniform brightness. In our experiments, we performed projector calibration by light attenuation. Using newer projector designs with light redirection [Damberg et al. 2016], we can achieve $20 \%$ higher gain per screen (dashed lines in Figure 10). The improvement in brightness is related to the audience size. The larger the audience, the lower the average gain of a directional screen.

Finally, in Figure 15, we present a comparison of images displayed on directional and matte screens. To generate these results we project an image onto the screen and capture its reflections at three points in the audience: the left corner, center, and the right corner of the middle row. We can see that our directional screens are significantly brighter. Moreover, the image brightness is consistent across the whole audience. Please note that the middle image for the home theater screen is from a viewing location outside the considered audience. Therefore, the non-uniform brightness of the directional screen at this view is expected.

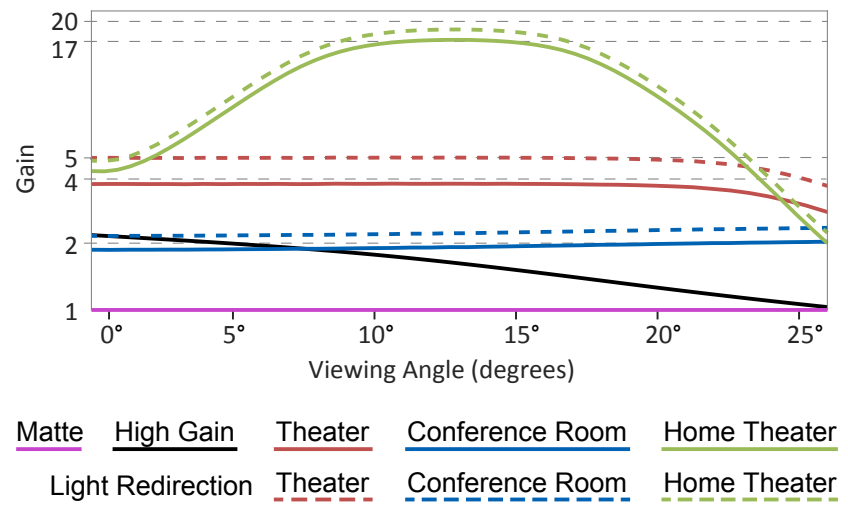

Figure 10: Brightness (gain) of each screen relative to the brightness provided by a matte screen. Gain is presented on a logarithmic scale which better corresponds to human perception. Solid lines represent our screens with calibration performed by light attenuation, dashed lines with a projector which redirects light [Damberg et al. 2016].

\subsection{Fabricated Prototypes}

In addition to our simulations, we also fabricated central fragments of our designs. Different techniques can be used to manufacture such surfaces, e.g., milling, 3D printing, embossing, etc. We chose milling as it offers high fabrication accuracy and a wide range of materials, including highly reflective ones such as aluminum. Due to the time requirements for milling the geometry on a nonprofessional device as well as the relatively small working volume of our machine, we were able to fabricate only small fragments of our theater and home cinema screens.

Milling the Geometry. We used a 3-axis milling machine, Roland EGX-600 with a 10 micron step resolution, for manufacturing of the prototypes (Figure 11). We chose hard aluminum, which was 
manually polished after milling to achieve mirror-like reflectance. Reproduction of small cavities in the final screen was challenging due to technical limitations (i.e., step and tool size) and some cavities could not be perfectly reproduced. For the validation we milled two prototypes (Figure 11). The first prototype has a size of $20 \times 20$ $\mathrm{cm}$ and corresponds to the theater directional screen. The second prototype has a size of $10.5 \times 7 \mathrm{~cm}$ and mimics the home theater split audience setup.

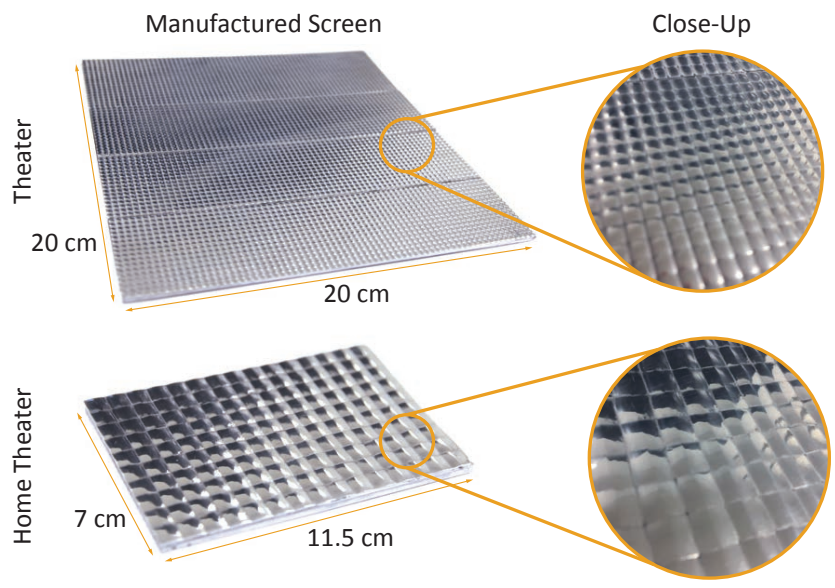

Figure 11: Photos of manufactured prototypes of a uniform and a split directional screen.

Capturing Setup. We have validated the fabricated prototypes by recreating our design setups. Two validation setups were used to evaluate screen reflection and audience coverage (Figure 12). To evaluate the audience coverage of a directional screen, we captured the shape of its reflection (Camera A), which should approximate the audience as closely as possible. The second setup evaluated the images provided to the viewers at different locations in the audience. To this end, we emulated a viewer moving through the audience while looking at the screen (Camera B). The photos were captured using a Nikon D750 camera. We used the following exposure settings to prevent overexposed regions in captured images: ISO 100 , shutter speed 1/30s and aperture F14.
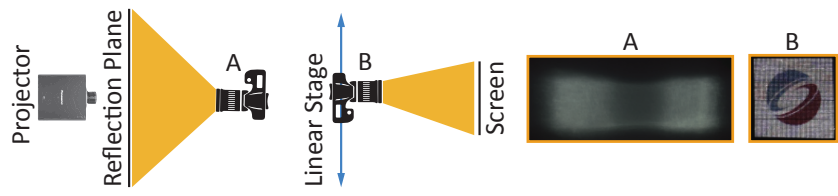

Figure 12: Prototype capture setup. A camera on the linear stage captures the reflection from the directional screen (B). A second camera captures the audience coverage of the examined directional screen (A).

Prototype Photo Results. Figure 13 shows results of the audience coverage test. We show, side by side, a simulation of the reflection and the actual reflection captured with our setup. We can see that

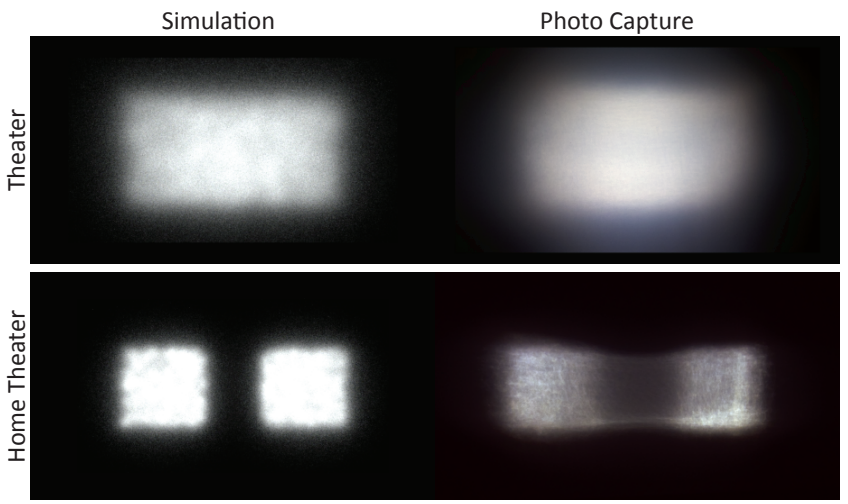

Figure 13: A directional screen reflection towards the audience. Simulations (left) are compared with fabricated prototypes (right).

our prototypes match the specified shape while providing light to the entire audience. A fraction of the reflected light bleeds outside the audience. We attribute this behavior to the BRDF of polished aluminum used in our simulations as well as manual polishing which may affect the accuracy of our geometries. For the home theater, there is also a reflection visible between the two audience volumes. This is caused by polishing, which removes sharp edges necessary for reproduction of perfect split audiences. We also evaluated the images produced by our screens as seen by viewers. To this end, we captured the illuminated screens from several viewing locations (Figure 14). As can be seen, our fabricated screen provides the highest brightness in the desired range (0-20 degrees), and becomes dimmer outside of this range.

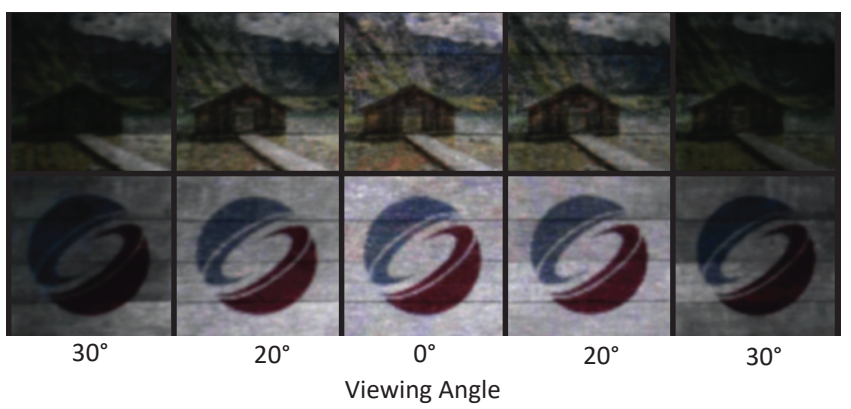

Figure 14: Manufactured prototype reflecting the SIGGRAPH logo and a cottage, viewed from various directions captured by camera B. According to the display specification, the reflection should be visible between 0-20 degrees and dark outside.

\section{DISCUSSION AND LIMITATIONS}

Brightness Gain. The brightness gain of directional screens depends on the audience size. The advantage when compared to a high-gain screen is uniformness of the reflected light. For large audience sizes, a high-gain screen might achieve higher peak brightness. However, this peak will quickly diminish as one moves away from 
the center of the audience. A directional screen is capable of providing a uniform brightness across the whole audience.

Fabrication Limitations. Using our low-cost manufacturing technique, a perfect reproduction of our designs was challenging. Our milling machine could not reproduce small cavities. Also, the manual polishing affected the accuracy of the geometry. This is manifested as uneven brightness of the physical prototypes (Figure 14) which was not present in our simulated results (Figure 15). Our surfaces also have a small diffuse component. This compensates for a limited number of microgeometry facets, but also causes some light bleeding on the boundaries of the audience.

Our fabrication technique is not ideal for large-scale mass production. We believe that embossing and coating the surface with aluminum pigments, similarly to [Coleman and Sharp 2013], can be used for commercial purposes. Since cinema chains provide standardized rooms, e.g., a standard IMAX screen is $22 \times 16.1 \mathrm{~m}$, and the audience remains similar across different venues, it is possible to split the screen into tiles and fabricate reusable embossing forms. Since our screen can be viewed as a surface with smoothly varying reflectance properties, small misalignments between the tiles as well as between the screen and the projector should not cause visible problems.

\section{CONCLUSION}

We presented an algorithm for generating directional projection screens. It improves on currently used high gain screens in two areas. First, our screens can achieve higher gain factors. Second, our design can eliminate the hot-spot effect which affects traditional high gain screens. These improvements will provide a better viewing experience and substantial energy savings for theaters. Besides a theoretical model, we also provide a validation using realistic simulation and fabrication techniques. We used polished aluminum which is cost-effective and relatively easy to tool. Even though we have a number of limitations regarding our fabrication technique, we have shown that our fabricated prototypes are more uniform and achieve higher brightness than current high gain screens. We believe that using more advanced manufacturing techniques can further improve the results and match the theoretical capabilities of our technique.

\section{ACKNOWLEDGMENTS}

This work was partially supported by European Research Council (ERC) grant 321135 CREATIV.

\section{REFERENCES}

Aleksandr D. Alexandrov. 2005. Convex Polyhedra. Springer.

CJ Bartleson and EJ Breneman. 1967. Brightness perception in complex fields. FOSA 57, 7 (1967), 953-957.

Glenn Berggren and Donald Carignan. 1967. On optimizing screen curvature in a front-projection indoor theater. Fournal of the SMPTE 76, 11 (1967), 1104-1106.

James F. Blinn. 1977. Models of light reflection for computer synthesized pictures. In ACM SIGGRAPH Computer Graphics.

JS Chandler and JJ De Palma. 1968. High-brightness projection screens with high ambient light rejection. Fournal of the SMPTE 77, 10 (1968), 1012-1024.

Sanford Cobb, Terrence M Conder, and Dennis F Vanderwerf. 1980. Directional front projection screen. (1980). US Patent 4,206,969.

David A. Coleman and Gary D. Sharp. 2011. Polarization preserving front projection screen microstructures. (2011). US Patent App. 13/290,978.
David A. Coleman and Gary D. Sharp. 2013. 54.2: Invited paper: high efficiency polarization preserving cinema projection screens. In SID Symposium Digest of Technical Papers.

Robert L. Cook and Kenneth E. Torrance. 1982. A reflectance model for computer graphics. ACM Trans. Graph. 1, 1 (1982), 7-24.

Matt Cowan. 2008. REALD 3D Theatrical System. A Technical Overview. In European Digital Cinema Forum.

Crystal Screens. 2016. Crystal Screens. (2016). www.crystalscreens.com.

Gerwin Damberg, James Gregson, and Wolfgang Heidrich. 2016. High brightness HDR projection using dynamic freeform lensing. ACM Trans. Graph. 35, 3 (May 2016), 24:1-24:11.

Gerwin Damberg and Wolfgang Heidrich. 2015. Efficient freeform lens optimization for computational caustic displays. Optics express 23, 8 (2015), 10224-10232.

Harkness Screens. 2014. http://www.harkness-screens.com/2d-3d-overview.html. (2014). Last accessed: January 2016.

Eric Heitz. 2014. Understanding the masking-shadowing function in microfacet-based BRDFs. Journal of Computer Graphics Techniques 3, 2 (2014), 32-91.

Bernard S. Hockley and Romauld Pawluczyk. 1991. Chromatically corrected directional diffusing screen. (1991). US Patent 5,046,793.

Reynald Hoskinson, Boris Stoeber, Wolfgang Heidrich, and Sidney Fels. 2010. Light reallocation for high contrast projection using an analog micromirror array. ACM Trans. Graph. 29, 6 (2010), 165:1-165:10.

ISO 3640-1976 1978. Cinematography. Television usage. Specification for motion-picture prints and sound records for international exchange of television programmes. Standard. International Organization for Standardization, Geneva, $\mathrm{CH}$.

Yanxiang Lan, Yue Dong, Fabio Pellacini, and Xin Tong. 2013. Bi-scale appearance fabrication. ACM Trans. Graph. 32, 4 (2013), 145:1-145:12.

Anat Levin, Daniel Glasner, Ying Xiong, Frédo Durand, William Freeman, Wojciech Matusik, and Todd Zickler. 2013. Fabricating BRDFs at high spatial resolution using wave optics. ACM Trans. Graph. 32, 4 (2013), 144:1-144:14.

Alfred Lit. 1959. Depth-discrimination thresholds as a function of binocular differences of retinal illuminance at scotopic and photopic levels. FOSA 49, 8 (1959), 746-751.

James J. Little. 1983. An iterative method for reconstructing convex polyhedra from Extended Gaussian Images. In Proc. of AAAI-83. Washington, DC, 247-250.

Luminit. 2016. www.luminitco.com. (2016). Accessed: 2016-04-08.

Tom Malzbender, Ramin Samadani, Steven Scher, Adam Crume, Douglas Dunn, and James Davis. 2012. Printing reflectance functions. ACM Trans. Graph. 31, 3, Article 20 (June 2012), 11 pages. https://doi.org/10.1145/2167076.2167078

Wojciech Matusik, Boris Ajdin, Jinwei Gu, Jason Lawrence, Hendrik P. A. Lensch, Fabio Pellacini, and Szymon Rusinkiewicz. 2009. Printing spatially-varying reflectance. ACM Trans. Graph. 28, 5 (2009), 128:1-128:9.

Hermann Minkowski. 1989. Ausgewählte arbeiten zur zahlentheorie und zur geometrie: Mit D. Hilberts Gedächtnisrede auf H. Minkowski, Göttingen 1909. Springer Vienna, Vienna, Chapter Allgemeine Lehrsätze über die konvexen Polyeder, 121-139.

Gustavo Patow and Xavier Pueyo. 2005. A survey of inverse surface design from light transport behavior specification. In Computer Graphics Forum.

RPC Photonics. 1980. www.rpcphotonics.com. (1980). Accessed: 2016-04-08.

Martin Richards and Dave Schnuelle. 2010. The effective gain of a projection screen in an auditorium. SMPTE Motion Imaging fournal 119, 7 (2010), 62-67.

Olivier Rouiller, Bernd Bickel, Wojciech Matusik, Marc Alexa, and Jan Kautz. 2013. 3D printing spatially varying BRDFs. IEEE Computer Graphics and Applications 33 (2013), 48-57.

Miller Schuck and Gary Sharp. 2012. 3D digital cinema technologies. Fournal of the Society for Information Display 20, 12 (2012), 669-679.

Jae Chul Shin, Hirohisa Yaguchi, and Satoshi Shioiri. 2004. Change of color appearance in photopic, mesopic and scotopic vision. Optical review 11, 4 (2004), 265-271.

Bolin Sun. 2001. Front ptojection screen. (2001). WO Patent 2,001,033,298.

James Tompkin, Simon Heinzle, Jan Kautz, and Wojciech Matusik. 2013. Contentadaptive lenticular prints. ACM Trans. Graph. 32, 4 (2013), 133.

Kenneth E. Torrance and Ephraim M. Sparrow. 1967. Theory for off-specular reflection from roughened surfaces. JOSA 57, 9 (1967), 1105-1112.

Johannes C Van De Ven. 1990. Front projection screen. (1990). US Patent 4,911,529.

Bruce Walter, Stephen R. Marschner, Hongsong Li, and Kenneth E. Torrance. 2007. Microfacet models for refraction through rough surfaces. In Proceedings of the 18th Eurographics Conference on Rendering Techniques. Eurographics Association, 195-206.

Tim Weyrich, Pieter Peers, Wojciech Matusik, and Szymon Rusinkiewicz. 2009. Fabricating microgeometry for custom surface reflectance. ACM Trans. Graph. 28, 3 (2009), 32:1-32:6.

Hongzhi Wu, Julie Dorsey, and Holly Rushmeier. 2013. Inverse bi-scale material design. ACM Trans. Graph. 32 (2013), 163:1-163:10. 

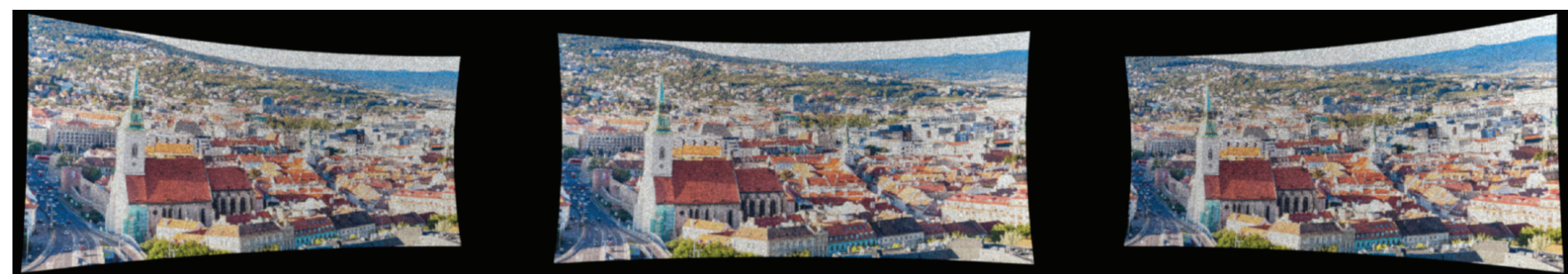

\section{Theater Directional Screen}
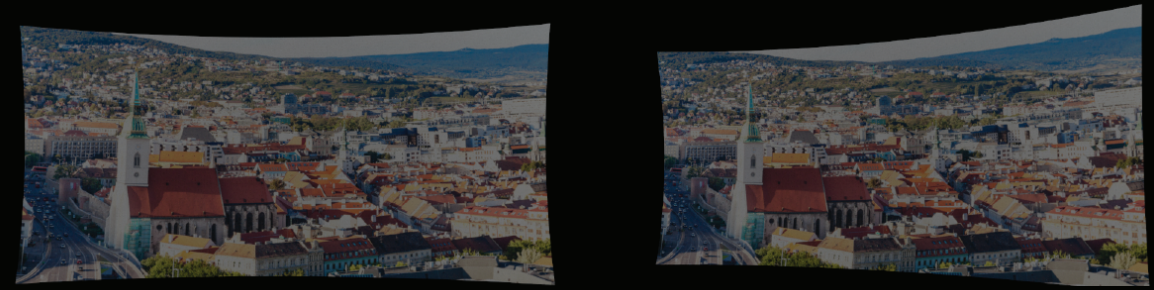

\section{Theater Matte Screen}
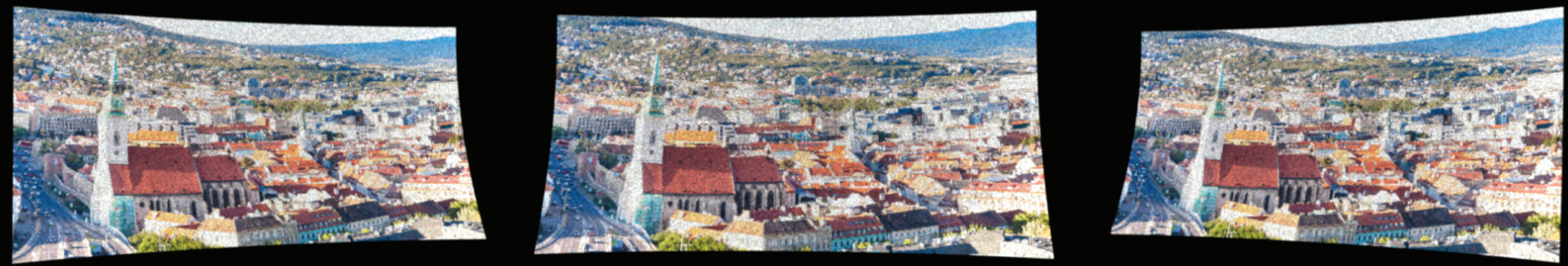

\section{Conference Room Directional Screen}
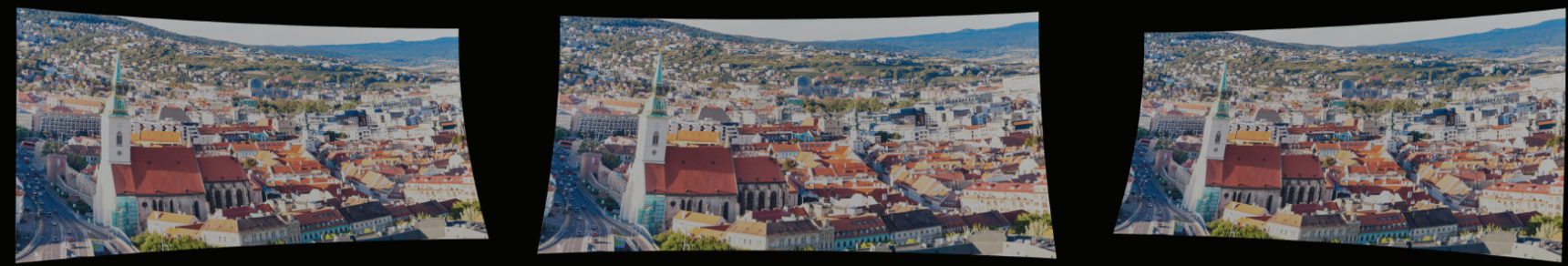

\section{Conference Room Matte Screen}
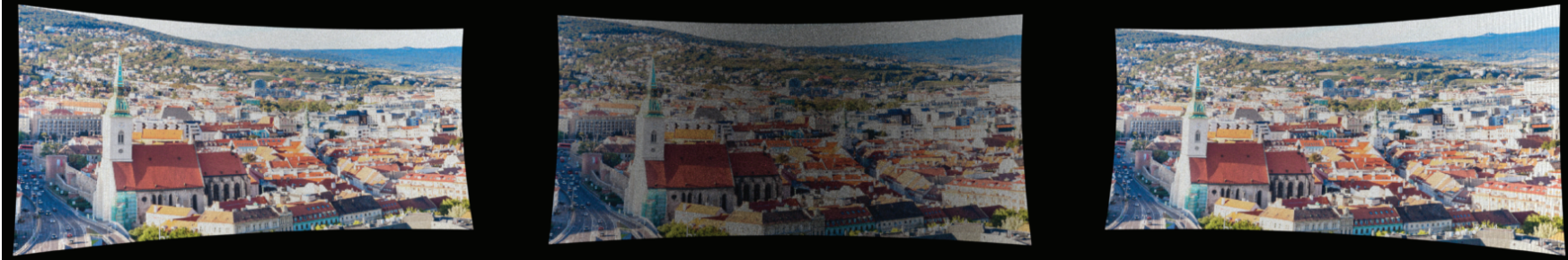

Home Theater Directional Screen

\section{Home Theater Matte Screen}

Figure 15: Renderings of an image projected on a directional screen and a matte screen. Please note that the middle image for the home theater screen is from a viewing location outside the considered audience. Therefore, the non-uniform brightness for the directional screen can be observed for this view. 